EPJ Web of Conferences 38, 14002 (2012)

DOI: $10.1051 /$ epjconf/20123814002

(c) Owned by the authors, published by EDP Sciences, 2012

\title{
Exotic nuclei and astrophysics
}

\author{
Yu. Penionzhkevich \\ Joint Institute for Nuclear Research, Dubna, Russia
}

\begin{abstract}
In recent years, nuclear physics investigations of the laws of the microscopic world contributed significantly to extension of our knowledge of phenomena occurring in the macroscopic world (Universe) and made a formidable contribution to the development of astrophysical and cosmological theories. First of all, this concerns the expanding universe model, the evolution of stars, and the abundances of elements, as well as the properties of various stars and cosmic objects, including "cold" and neutron stars, black holes, and pulsars. Without claiming to give a full account of all cosmological problems, we will dwell upon those of them that, in my opinion, have much in common with nuclear-matter properties manifesting themselves in nuclear interactions.
\end{abstract}

\section{Nucleosynthesis}

In the course of evolution, the Universe is permanently enriched in ever heavier chemical elements [1]. The abundances of chemical elements in the Universe is determined by various methods: by the spectrum of radiation from stars and by an element analysis of terrestrial and cosmic samples (meteorites and lunar samples). The curve of element abundances has the maxima for silicon and iron groups, whereupon it splits into branches: one includes neutron rich isotopes and has three doubled peaks near the magic numbers of $N=50$, 82 , and 126, while the second includes proton-rich isotopes, which are less abundant. The production of ${ }^{12} \mathrm{C}$ was one of the steps of nucleosynthesis. It was shown that carbon can be produced in the reaction ${ }^{4} \mathrm{He}+{ }^{8} \mathrm{Be} \rightarrow$ ${ }^{12} \mathrm{C}+\gamma$. However, the ${ }^{8} \mathrm{Be}$ nucleus is unstable with respect to decay to two alpha particles and lives $10^{-16} \mathrm{~s}$. Meanwhile, there is yet another possibility: at temperatures close to $10^{8} \mathrm{~K}$ and densities of about $10^{5}$ $\mathrm{g} \cdot \mathrm{cm}^{-2}$, three ${ }^{4} \mathrm{He}$ nuclei may form a ${ }^{12} \mathrm{C}$ nucleus via the two-step reaction ${ }^{4} \mathrm{He}+{ }^{4} \mathrm{He} \rightarrow{ }^{8} \mathrm{Be}+{ }^{4} \mathrm{He} \rightarrow{ }^{12} \mathrm{C}+\gamma$, its second step having a resonance character. This reaction makes it possible to explain the existence of carbon and other observable isotopes together with it. At each nuclear fusion step initiated by explosions of the outer envelope of stars, there occurs the formation of ever heavier nuclei: ${ }^{4} \mathrm{He},{ }^{12} \mathrm{C},{ }^{16} \mathrm{O},{ }^{28} \mathrm{~S}$, and ${ }^{56} \mathrm{Fe}$. It should be recalled that production processes are accompanied by the decay of these nuclei. Therefore, isotopes that have the highest stability (maximum binding energy per nucleon) are under the most favorable conditions. These include nuclei in the region around $A \approx 60$. This explains an enhanced abundance of iron-group nuclei (for the most recent data). A somewhat different mechanism governs the production of nuclei heavier than iron -- this is the mechanism of sequential reactions of radiative neutron capture by elements of the iron group. The fact that the curve of element abundances features double peaks in the curve of abundances of elements [2] suggests the occurence of two different neutron-capture processes-these are so-called $r$ and $s$ processes, which correspond to different neutron densities. In the case of low neutron densities [ $\boldsymbol{s}$ (slow) process], the radiative capture process $(A, Z)+n \rightarrow(A+1, Z)+\gamma$ leads to the production of an isotope of mass greater by unity than the mass of the target nucleus. If the $(A+1, Z)$ final nucleus is stable, radiative capture may also occur on it and lead to the production of a new isotope, $(A+2, Z)$. At low fluxes, the $(A+x, Z)$ nucleus decays before it absorbs a neutron. Predominantly, it decays via beta decay, which leads to the formation of a new nucleus, $(A+x, Z+1)$, or undergoes an entire chain of decays that ends up in a nucleus whose lifetime is sufficiently long for a new radiative capture process to have time to occur. This process is repeated many times, with the result that neutron-deficient nuclei of mass up to 200 are produced. After that, the nuclei undergo fission with a high probability, and this interrupts the $s$ process. At high neutron densities [ $\boldsymbol{r}$ (rapid) process], the $(A+x, Z)$ nucleus absorbs neutrons before undergoing decay, and this leads to new radiative capture processes. This proceeds until the decay chain reaches an isotope whose half-life is very short, and we arrive at the preceding case. Figure 1 shows the chains of $r$ and $s$ processes that lead to the production of various nuclei and isotopes from the ${ }^{56} \mathrm{Fe}$ nucleus. An enhanced abundance of the $N=50,82$, and 126 isotopes confirms the existence of $r$ and $s$ processes. It was shown 


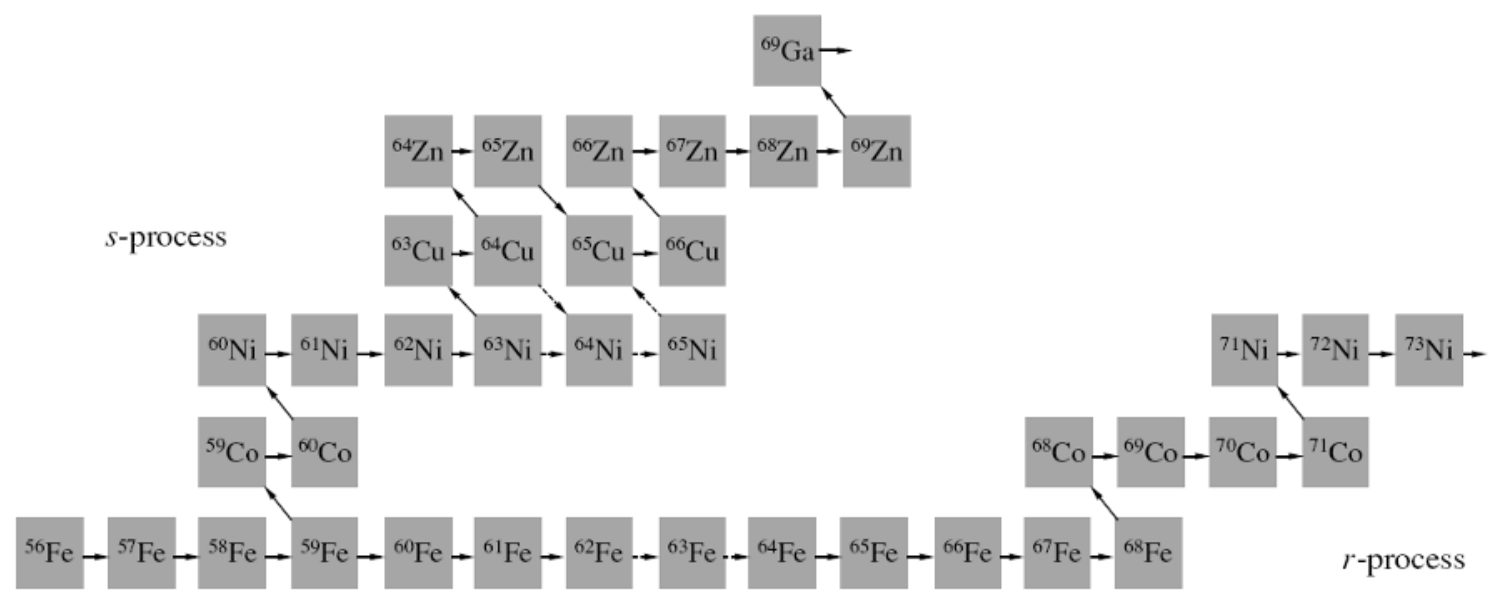

Fig.1. Chains of fast and slown eutron capture ( $r$ and $s$ processes) for the ${ }^{56} \mathrm{Fe}$ nucleus. The horizontal arrows, arrows at an angle of $135^{\circ}$, arrows at an angle of $-45^{\circ}$, and dashed-line arrows, indicate, respectively, $n$ capture, $\beta$ - decay, $\beta+$ decay, and alternative channels of ${ }^{65} \mathrm{Cu}$ production.

experimentally that the abundance of elements is in inverse proportion to the total neutron-capture cross sections. For nuclei characterized by magic numbers, these cross sections are several orders of magnitude smaller than the respective cross sections for other neighboring nuclei. From the point of view of nuclear physics, this result is a manifestation of magic numbers. For astrophysics, this proves the existence of the $S$ process. Also, the existence of the $Z=114$ shell and, accordingly, the enhancement of stability of superheavy nuclei in the vicinity of the doubly magic nucleus ${ }^{298} 114$ (114 protons and 184 neutrons) were also predicted. Calculations on the basis of the shell model revealed the possible existence of this superheavy nucleus with a halflife of up to about $10^{8} \mathrm{yr}[3]$.

\section{Nuclear physics experiments and astrophysics}

From the foregoing, it follows that energy deposition processes in the formation and explosion of stars and nucleosynthesis - they refer to the most fundamental problems in astrophysics - are directly related to particle physics and to nuclear physics investigations of effective reaction cross sections, masses of nuclei, and their lifetimes with respect to various modes of decay.

Nuclear reactions involving the capture of protons, neutrons, or alpha particles by various nuclei, including unstable ones, play an important role in nucleosynthesis processes. It is difficult to determine experimentally the rate of such reactions. The cross section for such processes also depends strongly on the temperature of the object. In the non-explosive evolution of a star, its temperature is relatively low, in which case the effective reaction cross section ranges between a few $\mathrm{pb}$ and a few $\mathrm{nb}$ units $\left(10^{-36}-10^{-33} \mathrm{~cm}^{2}\right)$. In the case of the explosion of stars, the temperature is very high (about $10^{9} \mathrm{~K}$ ), in which case the effective reaction cross section reaches a few mb units $\left(10^{-27} \mathrm{~cm}^{2}\right)$. In order to simulate such processes under laboratory conditions, we must have in our disposal a broad range of radioactive nuclei, which play a dominant role in the explosion of stars. In recent years, such a possibility appeared owing to the advent of accelerators for beams of radioactive nuclei. With the aid of such beams, one studies features of nuclear reactions, employing targets from hydrogen and helium. Below, we give some examples of such reactions. In recent years, astrophysicists vigorously discussed the existence of inhomogeneities in the distribution of hadron matter after the quark-hadron phase transition. In this case, the abundance of $A \approx 7$ elements can be well above the predictions of the standard model. In an inhomogeneous process of this kind ("big bang"), reactions like ${ }^{8} \mathrm{Li}(\alpha$, $n)^{11} \mathrm{~B}$ and ${ }^{6} \mathrm{He}(\alpha, n){ }^{9} \mathrm{Be}$ are of crucial importance for nucleosynthesis. In simulating the hot proton-proton channel, which may occur in the explosion of supernovae, it is of interest to measure the rate of reactions like ${ }^{7} \mathrm{Be}(p, \gamma){ }^{8} \mathrm{~B},{ }^{7} \mathrm{Be}(p, \gamma){ }^{9} \mathrm{C},{ }^{8} \mathrm{Be}(p, \gamma){ }^{9} \mathrm{C}$, and ${ }^{11} \mathrm{C}(p, \gamma){ }^{12} \mathrm{~N}$. Another, indirect, means for determining reaction rates and for studying nucleosynthesis consist in obtaining, by nuclear physics methods, spectroscopic data on nuclei far from the drip line. These data are used directly to describe $s$ and $r$ processes. High precision knowledge of the properties of decays of nuclei at the socalled turning points of the $r$ process is of paramount importance here. In Ref. [4], the scheme of fast neutron capture in the region of $\mathrm{S}, \mathrm{Cl}$, and Ar nuclei was obtained on the basis of data from the joint Dubna (Russia)GANIL (France)-Mainz (Germany) experiment in heavyion-accelerator beams. The half-lives $\left(T_{1 / 2}\right)$ and neutronic decay probabilities $\left(P_{n}\right)$ were measured in those experiments to a high precision for the neutron-rich isotopes of $\mathrm{S}, \mathrm{Cl}$, and Ar elements. The maximum time of irradiation with neutrons, the neutron flux, and the corresponding temperature of the star emitting neutrons on the time scale were calculated in accordance with this scheme (figure 2).

The discovery and investigation of new types of radioactive decay (beta-delayed fission, proton decay, shape isomerism) are among the last successes of nuclear 


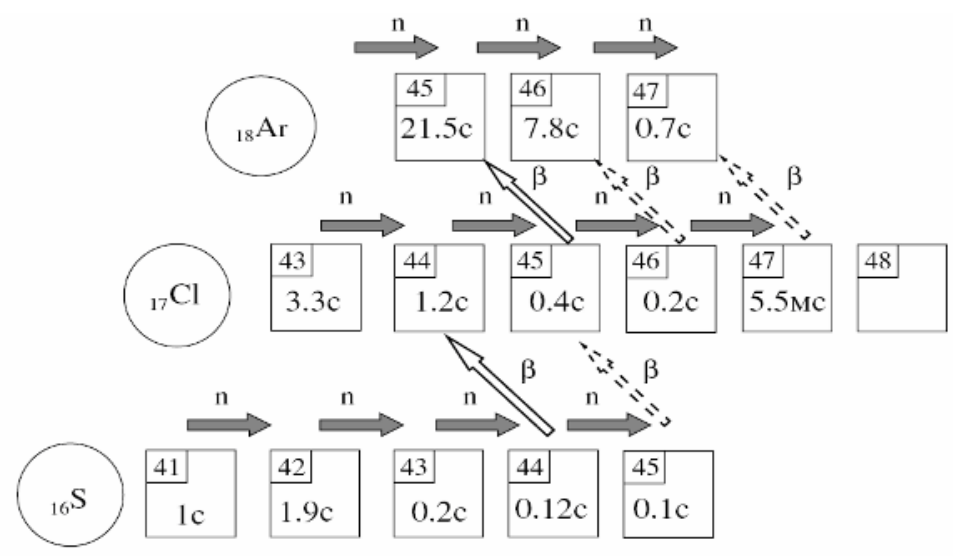

Fig. 2. Neutron capture path in the ${ }_{16} \mathrm{~S}$ to ${ }_{18} \mathrm{Ar}$ chains for stellar temperature of $\mathrm{T}=8.10^{8} \mathrm{~K}$ and neutron density $5.10^{-5} \mathrm{~mol} \mathrm{~cm}^{-3}$ with our experimental data [4]. At both $\mathrm{N}=28$ "turning point" isotopes, ${ }^{44} \mathrm{~S}$ and ${ }^{45} \mathrm{Cl}, \beta$ - decay back to stability starts to dominate over further neutron capture. Hence, the possible $\mathrm{A}=46,47$ progenitors of ${ }^{48} \mathrm{Ca} /{ }^{46} \mathrm{Ca}$ ratios can be obtained, as required to explain the observed abundances in EK-1-4-1 meteorite.

physics. The inclusion of these decay forms has a substantial effect on the calculation of the nuclideproduction chain and is an important problem that relates nuclear physics and astrophysics. Reactions involving loosely bound nuclei and proceeding at energies close to the Coulomb barrier are of particular interest for astrophysics. These reactions have many specific features, which were discovered in recent years by using beams of radioactive nuclei. An enhancement of interaction cross sections in the subbarrier energy region is one of such features (figure 3 ).

This effect manifests itself most strongly for cluster nuclei $\left({ }^{6,9} \mathrm{Li}\right)$ and for nuclei involving a neutron halo $\left({ }^{6} \mathrm{He}\right)[6,7]$. Deep-inelastic transfer reactions, breakup reactions, and complete-fusion reactions are dominant interaction channels for such nuclei. The breakup process followed by the fusion of the residual nucleus has been the subject of numerous theoretical and experimental studies. In accordance with classical ideas, the fusion of interacting nuclei occurs after they overcome the barrier that is determined by the long range Coulomb forces and the repulsive component of the short-range nuclear potential. However, the fusion process has a more complicated character in the case of the interaction between loosely bound nuclei, because, with a high probability, such nuclei may undergo breakup followed by the capture of a residual nucleus (incomplete fusion).

This complicates substantially the description of interaction in such systems and leads to new, unexpected, effects at energies in the vicinity of the Coulomb barrier, such as deep subbarrier fusion and reactions of cluster transfer from loosely bound nuclei, which, as a rule, have a cluster structure. By way of example, we indicate that, in the reaction of neutron transfer in case of interaction of ${ }^{6} \mathrm{He}$ nuclei, the cross section reaches a value of several barns and has a maximum in the vicinity of the Coulomb barrier. A large cross section for the transfer of one neutron and its smooth decrease toward the region of low energies (to $5 \mathrm{MeV}$ ) may be indicative of the mechanism of interaction between a quasifree neutron of the ${ }^{6} \mathrm{He}$ nucleus with the target nucleus. A similar example is well known for $(d, p)$ reactions. Here, in the deep subbarrier
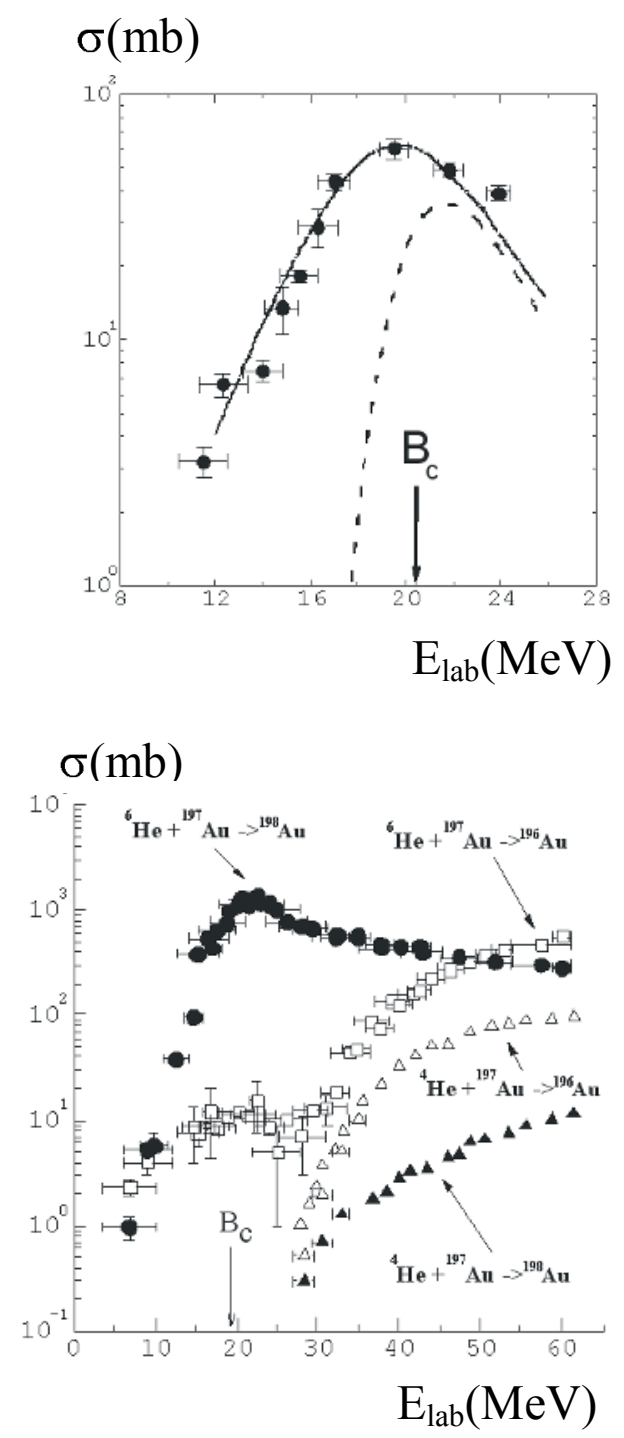

Fig. 3. Excitation functions for the reactions (close boxes) ${ }^{206} \mathrm{~Pb}$ $\left({ }^{6} \mathrm{He}, 2 n\right){ }^{210} \mathrm{Po}$ (a). The dashed curves are the results of statistical-model calculations. Solid curve stands for the calculations within the sequential-fusion model [5]. Excitation function for ${ }^{197} \mathrm{Au}+{ }^{6} \mathrm{He}$ reactions (b) leading to the production of the isotopes (closed boxes) ${ }^{196} \mathrm{Au}$ and (open circles) ${ }^{198} \mathrm{Au}$. Here, $B_{c}$ is the Coulomb barrier for ${ }^{6} \mathrm{He}+{ }^{197} \mathrm{Au}$ reactions. 
region, there is a significant increase in the cross section (so-called Oppenheimer-Phillips resonance) because of the polarization of a loosely bound deuteron. In our case, this effect may be more pronounced because of a lower binding energy for the ${ }^{6} \mathrm{He}$ nucleus than for the deuteron and because of stronger Coulomb forces of repulsion of alpha particles in the ${ }^{6} \mathrm{He}$ nucleus in relation to the proton in the deuteron. At the present time, there are several theoretical approaches attempting to describe interactions between loosely bound nuclei. Difficulties in describing them stem from the need for employing theories that take into account the interaction of several nuclear fragments. It is of importance to obtain deeper insight into the mechanism of such reactions, especially with allowance for astrophysical aspects of such processes involving light nuclei at low energies. An interesting result was obtained recently on the basis of an analysis of excitation functions for deep inelastic transfer reactions on ${ }^{6} \mathrm{Li}$ nuclei [8]. This analysis revealed that the capture of a deuteron from ${ }^{6} \mathrm{Li}$ by the target nucleus is a dominant mechanism for this reaction. Thus, the excitation of the projectile nucleus in the field of the target nucleus and its polarization simplify the mechanism of deuteron capture, the Coulomb barrier for it being lower than in the case of alpha-particle capture. This mechanism is peculiar to the majority of loosely bound nuclei that have a cluster structure. The above results are of paramount importance for solving astrophysics problems - in particular, for obtaining deeper insight into the mechanism of production of light elements in the Universe. In nucleosynthesis, a large cross section for the interaction of cluster loosely bound nuclei $\left({ }^{6} \mathrm{He},{ }^{9} \mathrm{Li},{ }^{7} \mathrm{Be}\right.$, etc) may change the chain of beta decays leading to the production of various elements and, hence, the whole scenario of nucleosynthesis [9]. In view of this, the following reaction channels may prove to be the most probable for the synthesis of light stable nuclei: ${ }^{1} \mathrm{H}\left({ }^{6} \mathrm{He}, n\right)^{6} \mathrm{Li}$, ${ }^{12} \mathrm{C}\left({ }^{6} \mathrm{He}, 2 n\right){ }^{16} \mathrm{O},{ }^{1} \mathrm{H}\left({ }^{9} \mathrm{Li}, n\right){ }^{9} \mathrm{Be},{ }^{3} \mathrm{He}\left({ }^{9} \mathrm{Li}, 2 n\right){ }^{10} \mathrm{~B}$, and so on.

One cannot help mentioning neutrino astrophysics as yet another aspect of nuclear astrophysics. Academician B. Pontecorvo wrote that nowhere does the relationship between the microscopic world and cosmos manifest itself as clearly as in neutrino physics. A determination of the astrophysical factor for the reaction involving the production of the ${ }^{8} \mathrm{~B}$ nucleus is one of the interesting problems related to the production of solar neutrinos. This nucleus is a source of high-energy neutrinos produced in solar processes. So far, calculations dealing with high energy solar neutrinos underestimate substantially the fluxes of such neutrinos in comparison with experimentally observed fluxes. The structure of ${ }^{8} \mathrm{~B}$ is unusual in what is concerned with the distribution of the proton and neutron densities. This nucleus features a proton halo - as was compellingly shown in [10], the rms radius of the proton distribution of this exotic nucleus is larger than the rms radius of the neutron distribution: $\left\langle r_{p}^{2}>-<r_{n}^{2}\right\rangle=0.49 \mathrm{fm}$; the cross section for its interaction with other nuclei differs substantially from what one would expect for the interaction of ordinary nuclei. For the reaction ${ }^{7} \mathrm{Be}(p, \gamma)^{8} \mathrm{~B}$, the astrophysical factor defined as $S_{17} \sim 2.99 \times 10^{6} \rho(10 \mathrm{fm}) S$ (where $\rho$ is the density distribution and $S$ is the spectroscopic factor) and found from experiments studying the interactions of the ${ }^{8} \mathrm{~B}$ nucleus proved to be $25 \%$ larger than that predicted earlier. It should be noted that a beam of accelerated ${ }^{8} \mathrm{~B}$ ions was used in the experiments being discussed. Therefore, new generation of radioactive beam accelerating facilities also play an important role in the development of astrophysics studies. Only a few examples illustrating connections between nuclear physics and macroscopic physics have been given above. Despite the smallness of the number of particles involved in the formation of nuclei (not more 300), they constitute a unique system for simulating problems of the macroscopic world. Under laboratory conditions, nuclei that have unusual properties, so-called exotic nuclei (those that have a high temperature or a high angular momentum, those that consist of neutrons almost exclusively, and superdeformed nuclei), which can in principle be produced in cosmic processes, are obtainable by means of nucleus-nucleus collisions implemented at modern accelerators of heavy ions at energies of several hundreds $\mathrm{MeV} /$ nucleon. Figure 4 shows the temperature of a nucleus as a function of its excitation energy, which is proportional to the projectile energy. These data were obtained in three experiments: in Dubna (Russia) [11], at low energies, in CERN (Switzerland) at excitation energies in the range $2-8 \mathrm{MeV}$, and in Darmstadt (Germany) at energies in the range $8-15 \mathrm{MeV}$. One can see that, as the excitation energy increases in the range 2 $8 \mathrm{MeV}$, the nuclear temperature does not increase, but, at low energies, the temperature increases sharply, in just the same way as it does at very high energies. This sensational result demonstrates, first, that one may reach

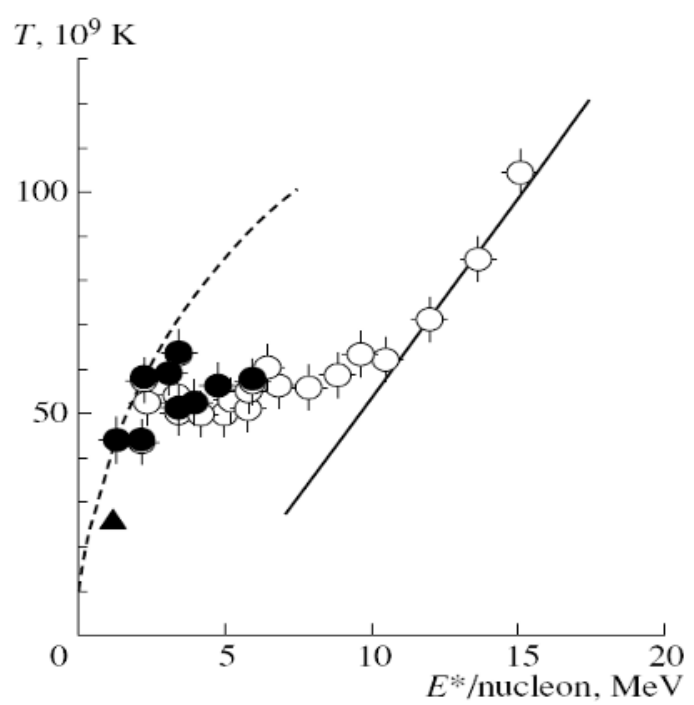

Fig. 4. Experimental dependences [(closed triangles) Joint Institute for Nuclear Research (Dubna), (open circles) GSI (Darmstadt, Germany), and (closed circles) CERN (Switzerland)] of the temperature $T$ of a nucleus on its excitation energy. The solid and dashed curves represent the dependences in question for a ideal gas and a liquid, respectively. The inflection points of the curves drawn through the experimental points correspond to the liquid-gas phase transition. 
in nuclear reactions temperatures that exists only at the instant of star formation and, second, confirms the occurrence of phase transitions in hot objects, which was assumed considering the expanding universe and the explosion of stars.

A simulation of processes leading to the formation of neutron stars is yet another example. Here, experimentalists try to approach as close as possible the neutron drip line and even to study the properties of nuclei beyond it (so-called quasi-stationary states of super-neutron-rich nuclei). Attempts to discover systems consisting of neutrons exclusively (tetraneutron) or one proton and several neutrons (hydrogen-6, hydrogen-7, and so on) were continued for many years [12]. In the programs for research centers involved in the construction of new generation of accelerator facilities, including accelerators with intense beams of radioactive nuclei, the production of new exotic nuclei and investigation of their properties with an eye to simulating processes proceeding in the Universe are among the main aims.

\section{Conclusions}

Our knowledge about the formation of the Universe, nucleosynthesis, and properties of new stellar formations is still being developed, and this development is tightly related to the accumulation of new data in the realms of nuclear and particle physics. Therefore, the derivation of information about the mechanisms of Universe formation and nucleosynthesis on the basis of present ideas of nuclear astrophysics is the main task in constructing a unified cosmological model.

\section{Acknowledgement}

This work was supported by the Russian Foundation for Basic Research (project no. 07-02-00271-a).

\section{References}

1. J. Narlikar, Violent Phenomena in the Universe (Oxford University, Oxford, New York, 1982; Mir, Moscow, 1985)

2. D.G. Baranov, et al. , Izv. Akad. Nauk., Ser. Fiz., 69, 832 (2005)

3. Yu.Ts. Oganessian, J. Mod. Phys. E 16, 949 (2007)

4. O. Sorlin, et al., Phys. Rev. C 47, 2941 (1993)

5. V.I. Zagrebaev, Phys. Rev.C 67, 061601 (R) (2003)

6. M. Dasgupta, et al., Phys. Rev. C 70, 024606 (2004)

7. Yu. E. Penionzhkevich, et al., Eur. Phys. J. A 31, 185 (2007)

8. Yu. Penionzhkevich, et al., J. Phys. G: Nucl. Part. Phys. 36, 025104 (2009)

9. V. Zagrebaev, et al., Phys. Rev. C 75, 035809 (2007)

10. F. Negoita, et al., Phys. Rev. C 54, 1787 (1996)

11. Yu. Penionzhkevich, Phys. At. Nucl. 73, 1460 (2010)

12. A. Ogloblin, Yu.E. Penionzhkevich, in Treatise on Heavy Ion Science, ed. D. Allan Bromley (Plenum Press, New York, 1989) p. 260. 